\title{
Que sait-on des précipitations en altitude dans les Andes semi-arides du Chili ?
}

\author{
Pierre-Yves BOURGIN ${ }^{1 *}$, Vazken ANDREASSIAN ${ }^{1}$, Simon GASCOIN ${ }^{2}$, Audrey VALERY ${ }^{3}$
}

\author{
1. Irstea, UR HBAN, BP 44, 92163 Antony Cedex :, e-mail : pierre-yves.bourgin@gdfsuez.com \\ *now at Tractebel Engineering - Coyne et Bellier \\ 2. CEAZA, Raul Bitran s/n, La Serena, Chili \\ CESBIO 18 Avenue Edouard Belin Bpi 2801, 31401 Toulouse Cedex 9, e-mail :simon.gascoin@cesbio.cnes.fr \\ 3. Tractebel Engineering - Coyne et Bellier, 5 rue du 19 Mars 1962, 92622 Gennevilliers Cedex, e-mail :audrey.valery@gdfsuez.com
}

RÉSUMÉ. - On cherche souvent à établir la distribution spatiale des précipitations sur une grille régulière. Les méthodes d'interpolation spatiale sont couramment utilisées pour estimer de telle distribution à partir des observations au sol d'un réseau de mesures. Dans les régions montagneuses, l'estimation des précipitations en altitude reste un défi et les résultats des interpolations spatiales doivent être contrôlés avec le maximum d'attention. Nous proposons dans cet article une méthodologie de validation d'un champ de précipitation à trois niveaux successifs. Elle est appliquée dans le contexte d'une région semi-aride montagneuse, le Norte Chico au Chili $\left(26^{\circ} \mathrm{S}-32^{\circ} \mathrm{S}\right)$. L'application de cette méthodologie a permis de mettre en évidence les bénéfices apportés par une méthode d'interpolation développée par Valéry [1] pour les zones de montagne. En particulier, le bilan hydrologique des bassins versants les plus en altitude est rendu plus vraisemblable.

Mots Clés : Précipitation, interpolation et extrapolation spatiale, altitude, correction altitudinale, validation, équivalent en eau, bilan hydrologique

\section{What do we know about high-altitude precipitation in the semi-arid Andes of Chile?}

ABSTRACT. - Mapping precipitations on a regular grid is often required for hydrological and ecological modelling. The spatial interpolation methods are generally used to estimate such a distribution from ground-based measurements. In the case of mountainous areas, the estimation of precipitation amounts is still a challenging task and the results of spatial interpolation should be verified as much as possible. Here we describe a three-steps method for the validation of a precipitation map. This is used in the context of a mountainous semi-arid region, the Norte Chico in Chile $\left(26^{\circ} \mathrm{S}-32^{\circ} \mathrm{S}\right)$. The implementation of this validation method showed the benefits of an interpolation method developed by Valéry [2010] for mountainous areas. The hydrological balance of the high-altitude watersheds is now more realistic.

Key words : Precipitation, spatial interpolation method, extrapolation, altitude, altitudinal corrections, validation, water equivalent, water balance

\section{INTRODUCTION}

\section{I.1. Comment valider un champ de précipitation ?}

Etablir la distribution spatiale de la précipitation sur une grille régulière est souvent nécessaire à la modélisation hydrologique et écologique. Lorsqu'on utilise les informations des observations au sol pour interpoler un champ de précipitation dans l'espace à deux dimensions $(\mathrm{x}, \mathrm{y})$, il est nécessaire de distinguer la validation en interpolation et la validation en extrapolation. Dans les régions de montagne, il faut prendre en compte une coordonnée supplémentaire, l'altitude (z). Ainsi, l'extrapolation spatiale désigne l'estimation de la précipitation en dehors des limites dans les trois dimensions de l'espace du polygone formé par les stations de mesure. L'estimation de la précipitation en extrapolation est a priori plus incertaine qu'en interpolation et doit donc être l'objet d'une validation rigoureuse. Par ailleurs, les zones de montagne posent des difficultés spécifiques quant-à l'estimation des précipitations :

- La forte variabilité spatiale due à la topographie complexe ;
- Le réseau de mesures est généralement moins dense en altitude [Viviroli D. and R. WeingartneR, 2004] : l'accès est difficile, les conditions sont rigoureuses ;

- Les précipitations solides sont mal mesurées. Mais l'accumulation de neige peut permettre de repousser la mesure de la hauteur de neige à un moment où les conditions sont favorables.

\section{I.2. Trois niveaux de validation}

Dans cet article, nous présentons trois niveaux successifs de validation d'une cartographie des précipitations. D'abord dans la zone d'interpolation [Lloyd, 2005], puis dans la zone d'extrapolation, et enfin à l'échelle des bassins versants. Cette méthodologie est appliquée dans le contexte d'une région semi-aride montagneuse, le Norte Chico au Chili $\left(26^{\circ} \mathrm{S}-32^{\circ} \mathrm{S}\right)$ (figure 1, voir section II.1). Sur cette région, des écarts entre les précipitations et les écoulements et les difficultés à boucler le bilan hydrologique des bassins d'altitude ont été constatés par Favier et al. [Favier et al. 2009]. Ainsi, l'accent est mis sur les zones d'altitude et l'amélioration 
de la vraisemblance des bilans hydrologiques à l'échelle régionale par l'utilisation d'une méthode d'interpolation développée par Valéry [2010].

\section{ZONE D'ÉTUDE ET DONNÉES}

\section{II.1. Zone d'étude}

La région du Norte Chico est une région montagneuse et semi-aride située au nord du Chili (figure 1). Région de transition entre la Vallée Centrale au climat de type méditerranéen et le désert d'Atacama, l'un des plus arides au monde, le Norte Chico est caractérisé par des gradients topographiques et climatiques prononcés. Seulement $200 \mathrm{~km}$ séparent la côte Pacifique de la frontière naturelle formée par les Andes avec une altitude pouvant dépasser les $5000 \mathrm{~m}$. La variabilité spatiale des précipitations est extrême, organisée selon deux composantes principales. D'abord selon la direction sud-nord : le long de la côte, la précipitation annuelle moyenne diminue de $250 \mathrm{~mm}$ à moins de $20 \mathrm{~mm}$. Puis, au second ordre, selon la direction ouest-est, celle du gradient topographique : la précipitation est globalement multipliée par trois dans la haute cordillère d'après les registres disponibles. Parallèlement, la température décroît avec l'altitude et $80 \%$ de la précipitation annuelle a lieu pendant les trois mois d'hiver. La ressource en eau repose donc avant tout sur la fonte saisonnière du manteau neigeux accumulé en altitude pendant les mois d'hiver. Les zones de peuplement et d'activité sont au contraire concentrées sur la côte et le long des vallées, où l'agriculture irriguée est répandue. D'un point de vue hydrologique, les zones de source de production d'eau sont donc séparées des zones de consommation.

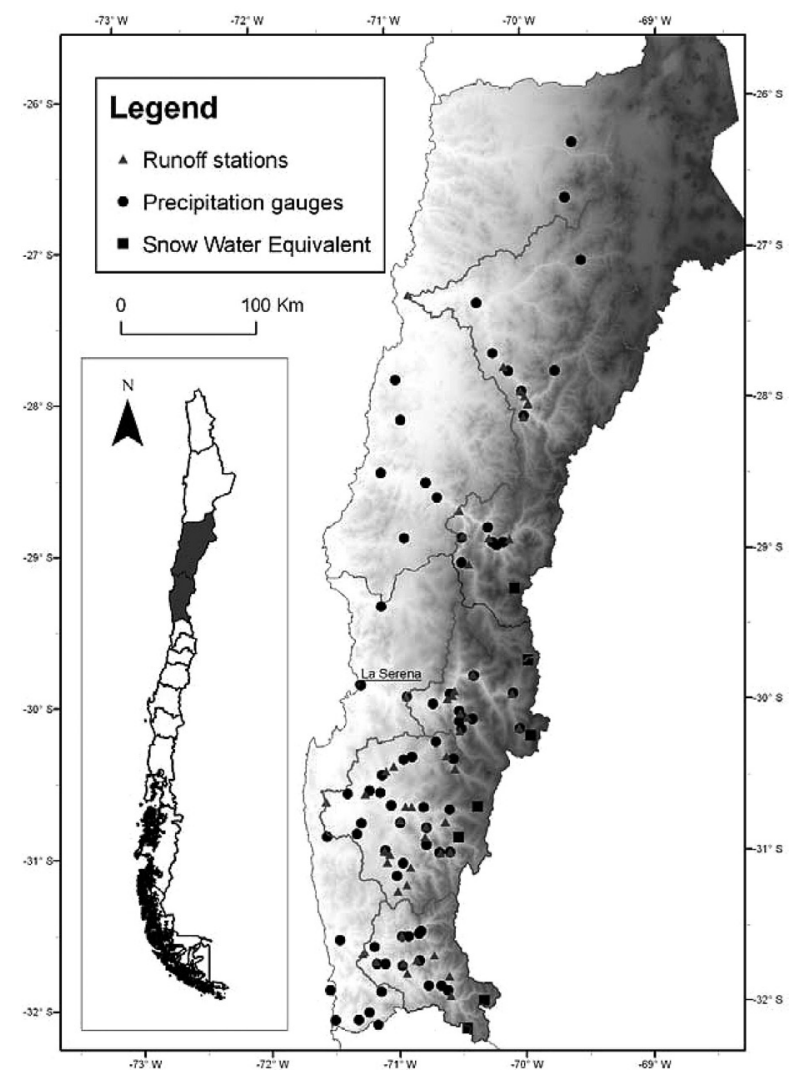

Figure 1 : Carte du Norte Chico présentant les stations de mesure et le gradient topographique

\section{II.2. Données}

Compte tenu des difficultés liées à l'estimation des précipitations en montagne (cf. I.1.), nous avons cherché à tirer le maximum d'information des données disponibles sur la zone d'étude. Ce faisant, une méthode de calcul et de validation visant à valoriser un jeu de données disparates a été mise en place.

\section{II.2.1. Stations de mesures de la précipitation et stations de jaugeage}

Les données de 75 stations de mesure de précipitation au sol et de 56 stations de jaugeage des débits, disponibles au pas de temps mensuel, ont été utilisées. La période commune retenue est 1975-2006. Les stations de mesure de précipitation sont majoritairement situées dans les zones de faible et moyenne altitude et dans les vallées. La plus élevée est située à $3100 \mathrm{~m}$, tandis qu'environ un tiers de la superficie totale du Norte Chico se trouve à une altitude supérieure. La densité du réseau de mesures est de $1500 \mathrm{~km}^{2}$ par station de mesures, tandis que l'Organisation Mondiale de la Météorologie préconise une densité de $250 \mathrm{~km}^{2}$ par station dans les zones montagneuses. Enfin, la possibilité de biais dans les mesures de débits est importante pour les stations de jaugeage situées en dessous de $1500 \mathrm{~m}$. Les écoulements naturels y sont en effet largement influencés par les aménagements hydrauliques (ouvrages de régulation, canaux d'irrigation) à des fins agricoles.

\section{II.2.2. Mesures de hauteur du manteau neigeux et équivalent en eau}

Des mesures en altitude de hauteur de neige et de densité ont été utilisées pour la validation du champ de précipitation en extrapolation dans les zones d'altitude. Ces mesures sont réalisées manuellement lors de sorties sur le terrain parfois appelées parcours nivométriques. La hauteur de neige est mesurée à intervalles réguliers le long d'un transect de plusieurs dizaines de mètres. La densité de la neige est également mesurée. En couplant les hauteurs moyennes et la densité moyenne mesurées, on obtient ainsi une hauteur d'eau liquide équivalente rendant compte de la lame d'eau précipitée. La limite de ce modèle d'équivalent en eau concerne la transformation de la neige et les fontes intermédiaires pouvant intervenir au cours de la saison hivernale et qui biaisent l'interprétation des hauteurs d'eau en lame d'eau écoulée. Les données utilisées pour cette étude sont directement des hauteurs d'eau équivalente qui proviennent de la Dirección General de Agua (Agence de l'eau chilienne). Elle a pour mission d'estimer les volumes d'eau accumulés en altitude et qui seront disponibles pour l'année. Ces estimations servent à anticiper le remplissage des barrages réservoirs et à l'attribution des quotas des droits à l'eau aux usagers. D'autres données proviennent des concessions minières installées en altitude pour lesquelles les chutes de neige impactent directement l'activité.

\section{II.2.3. Télédétection}

L'imagerie satellite du projet MODIS (NASA) a été utilisée pour suivre l'évolution saisonnière de la couverture neigeuse sur le Norte Chico. Le produit MOD10CM que nous avons utilisé est une estimation mensuelle binaire (neige/ absence de neige) de la présence de neige sur une grille de résolution $0,05^{\circ}$. 


\section{II.2.4. Modèle Numérique de Terrain (MNT)}

Un MNT de résolution $0,01^{\circ}$ couvrant la région du Norte Chico a été utilisé (source : Shuttle Radar Topography Mission).

\section{DISTRIBUTION SPATIALE DU CHAMP DE PRÉCIPITATION}

Une méthode innovante d'interpolation intégrant l'effet altitudinal développée par Valéry [2010] a été mise en œuvre. Les observations disponibles aux 75 stations de mesure de précipitation de la base de données servent à estimer au pas de temps mensuel la précipitation en chaque point d'une grille de résolution $0,01^{\circ}$ calquée sur le MNT. Cette méthode repose sur le voisinage et une correction multiplicative des lames écoulées basée sur la différence d'altitude $z$ entre un point cible de la grille et les stations de mesures voisines. Les équations (1) et (2) ci-dessous donnent l'estimation de la précipitation pour chaque point de la grille $x$ et pour chaque mois $m$ de la période 1975-2006. Le terme $w_{x, i}$ est le terme de pondération en inverse de la distance à la puissance $\alpha$. $N$ est le nombre total de stations de précipitations. $\beta_{a l t}$ est le facteur de correction altitudinale.

$$
\begin{gathered}
P_{m, x}^{s i m}=\frac{\sum_{i}^{N}\left[w_{x, i} \times P_{m, i}^{o b s} \times \exp \left[\beta_{a l t} \times\left(z_{x}-z_{i}\right)\right]\right]}{\sum_{i}^{N} w_{x . i}} \\
w_{i, j}=1 /\left(\text { distance }_{i, j}\right)^{\alpha}
\end{gathered}
$$

La méthode de validation croisée «Jack-Knife » a été utilisée. Elle consiste à extraire à tour de rôle chacun des points d'observation et à estimer la précipitation au point exclu à l'aide des stations restantes. En minimisant l'écart entre observation et reconstitution, on parvient à caler les paramètres $\alpha$ et $\beta$ du modèle.

De nombreuses tentatives d'amélioration ont été mises en œuvre. La complexification du modèle ne s'est pas révélée pertinente. La figure 2 présente la distribution spatiale de la précipitation moyenne annuelle calculée sur la période 1975-2006

\section{LA VALIDATION MULTI-NIVEAUX DU CHAMP DE PRÉCIPITATION}

Nous cherchons à présent à valider le champ de précipitation calculé au pas de temps mensuel. D'abord dans la zone d'interpolation où nous exploiterons les résultats de la validation croisée, c'est-à-dire les estimations aux points des stations d'observations, puis dans la zone d'extrapolation où nous tentons de valoriser les rares observations disponibles que sont les mesures de hauteur de neige. Enfin, la distribution sera contrôlée à l'échelle des bassins versants en testant le bouclage des bilans hydrologiques

\section{IV.1. $1^{\text {er }}$ niveau de validation : contrôle des estimations au droit des points de mesures}

La méthode de validation croisée donne, pour le couple optimal de paramètres, une chronique d'estimations de la précipitation mensuelle en chacune des 75 stations de mesure. Cette chronique est confrontée à la série des observations.

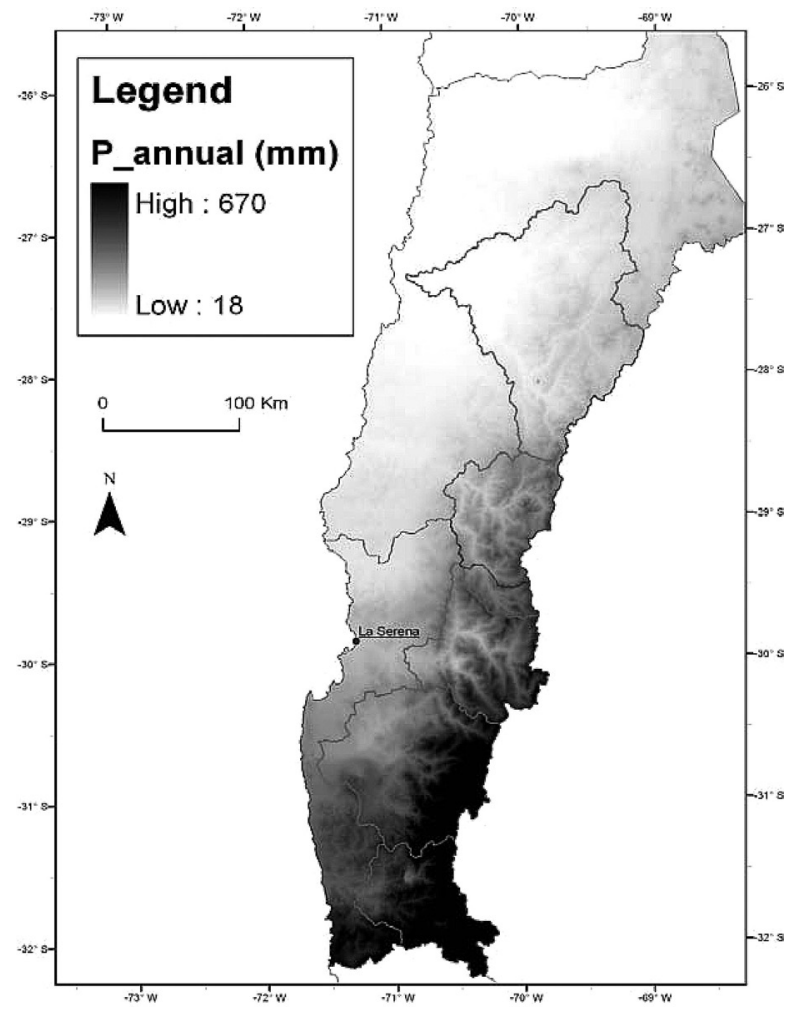

Figure 2 : Distribution spatiale de la précipitation moyenne annuelle calculée sur la période 1975-2006

La figure 3 ci-dessous présente les différences sur les précipitations moyennes annuelles observées et estimées. Une droite d'ajustement linéaire ainsi que la première bissectrice sont également représentées. Il n'existe pas de tendance générale à la sous-estimation ou la surestimation de la précipitation. On note toutefois que pour les plus forts cumuls, supérieurs à $200 \mathrm{~mm} / \mathrm{an}$, le modèle à davantage tendance à sous-estimer les cumuls. De plus, l'analyse spatiale du biais relatif, non reproduite ici, ne révèle pas de tendance régionale comme une dépendance à l'altitude ou à la latitude. Les biais principaux ont été analysés dans le détail. Ils s'expliquent principalement par la très grande variabilité des cumuls de précipitations observées qui peut exister entre des stations voisines et révèlent les limites inhérentes à toute interpolation. Enfin, la station de Los Moles qui présente le biais le plus important avec un facteur de 3 entre l'observation et l'estimation présente une série d'observation très incomplète de seulement 8 années et où les mois pluvieux d'hiver sont souvent

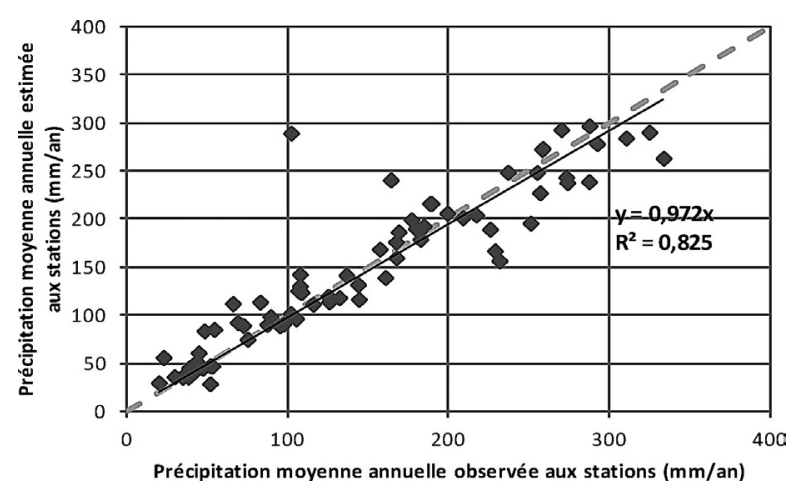

Figure 3 : Contrôle des précipitations annuelles moyennes estimées aux stations de mesure sur la période 1975-2006 
manquants (6 années sur 8). La pluie moyenne annuelle calculée pour l'observation est donc largement biaisée.

\section{IV.2. $2^{\text {ème }}$ niveau de validation :}

\section{la valorisation des mesures de hauteur de neige}

Il s'agit ici de contrôler dans la mesure du possible les estimations de précipitation en extrapolation, c'est-à-dire dans les zones de plus forte altitude. La valorisation des mesures de hauteur de neige n'est possible que dans un cadre d'hypothèses assez fortes et en gardant à l'esprit que les incertitudes sont conséquentes. C'est la fréquence des mesures de hauteur de neige qui fait principalement défaut puisque en moyenne, seule 1 à 2 mesures de hauteur de neige sont disponibles pour chaque hiver. Les 7 sites de mesures de hauteur de neige se trouvent à des altitudes supérieures à $3200 \mathrm{~m}$. Pour ces altitudes, la période entre mai et octobre est retenue comme période de précipitation solide. L'analyse porte sur les précipitations solides annuelles. Les équivalents en eau dérivés des mesures de hauteur de neige et densité sont confrontés à nos estimations, ainsi qu'aux résultats d'une interpolation spatiale classique de pondération par l'inverse de la distance (IDW en anglais) (sans prise en compte de l'effet altitudinal mais en utilisant la même procédure d'optimisation) (figure 4). A nouveau, pour nos estimations, il n'existe pas de tendance générale à la sous-estimation ou la surestimation. La variabilité annuelle est reproduite et la moyenne annuelle de précipitations solides est proche de l'observation. L'interpolation IDW a le même comportement mais sousestime constamment les précipitations. Le coefficient de corrélation et les biais relatifs moyens aux points d'observation sont donnés dans le Tableau 1. Ce résultat montre l'apport bénéfique de la correction altitudinale du modèle utilisé pour les zones les plus en altitude.
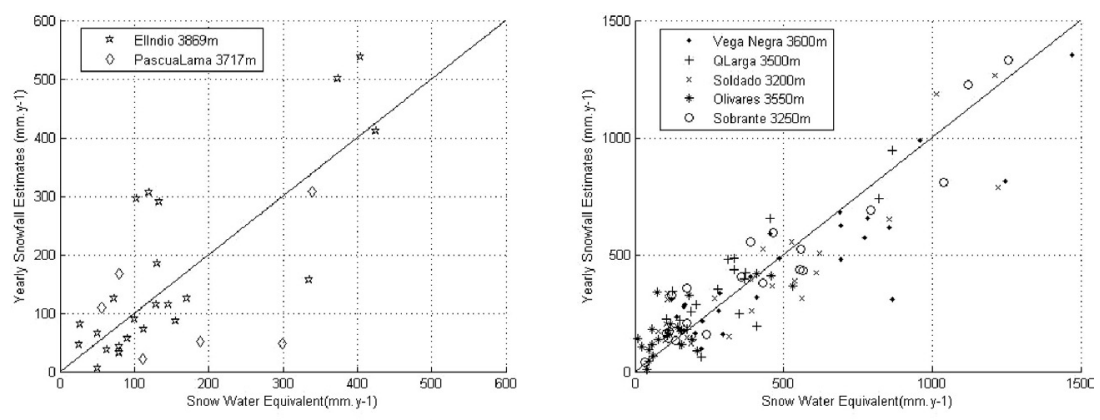

Méthode de Valéry [1]
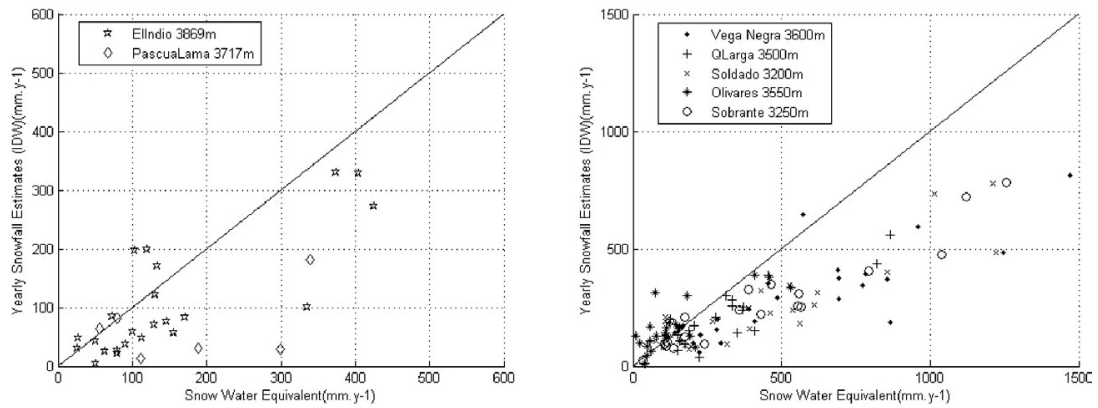

Méthode d'interpolation sans facteur de correction altitudinale

Figure 4 : Comparaison des estimations de chutes de neige. De bas en haut, on trouve respectivement les résultats issus de la méthode d'interpolation de Valéry, et d'une interpolation type IDW. Les données sont séparées en deux jeux : à gauche, les observations sont fournies par des compagnies minières et l'équivalent en eau annuel est calculé à partir de mesures mensuelles. A droite, les données proviennent de la DGA. L'équivalent en eau annuel est alors calculé comme le maximum des observations (maximum 1 à 2 observations par année).

Tableau 1 - Coefficient de corrélation et biais relatif moyen des précipitations neigeuses estimées

\begin{tabular}{|c|c|c|c|c|}
\hline & & & Valéry [1] & IDW \\
\hline $\begin{array}{c}\text { Coef. de corrélation } \\
\mathbf{R}^{2}\end{array}$ & & & $\mathbf{0 , 7 9 2 9}$ & $\mathbf{0 , 7 6 3 3}$ \\
\cline { 2 - 5 } & Nom & Alt (m) & \multicolumn{2}{|c|}{ Biais relatif moyen (\%) } \\
\hline 1 & El Indio & 3869 & 3,2 & $-33,5$ \\
\hline 2 & Pascua-Lama & 3717 & -34 & $-62,7$ \\
\hline 3 & Vega Negra & 3600 & $-8,5$ & $-44,9$ \\
\hline 4 & Quebrada Larga & 3500 & 13,3 & $-32,4$ \\
\hline 5 & Soldado & 3200 & $-7,9$ & $-43,3$ \\
\hline 6 & Olivares & 3550 & 22,1 & 11,1 \\
\hline 7 & Sobrante & 3250 & 4 & -39 \\
\hline
\end{tabular}




\section{IV.3. $3^{\text {ème }}$ niveau de validation : contrôle des bilans hydrologiques à l'échelle des bassins versants}

Il s'agit pour ce troisième niveau de validation de contrôler à l'échelle des bassins versants la vraisemblance des bilans hydrologiques. Une première étape consiste à calculer les coefficients d'écoulement, c'est-à-dire le ratio de l'écoulement annuel moyen (issu des mesures de débits) sur la précipitation annuelle moyenne. Ecoulement et précipitation sont calculés sur le même période de temps : la période de référence 1975-2006. L'agrégation de la précipitation à l'échelle d'un bassin versant a été réalisée par la moyenne des précipitations des mailles intersectant le bassin versant et pondérée par la fraction de la maille dans le bassin versant. Tous les coefficients d'écoulement obtenus sont inférieurs à la limite physique de $100 \%$. Cela semble trivial mais ce résultat révèle une amélioration de la vraisemblance des bilans hydrologiques par rapport aux travaux de Favier et al. [2009] qui rapportaient des coefficients d'écoulement apparents obtenus par une autre technique d'interpolation supérieurs à $100 \%$, (figure 5). Nous avons constaté que les estimations de précipitations obtenues grâce à la méthode d'interpolation de Valéry [2010] sont plus performantes que celles obtenues par Favier et al. [2009], particulièrement en altitude. Ceci s'explique par l'intégration d'un facteur de correction altitudinale et l'optimisation des paramètres du modèle d'interpolation de Valéry [2010].

Afin de poursuivre la validation du champ de précipitation, l'équation suivante du bilan hydrologique du bassin versant est utilisée :

$$
\mathrm{Q}=\mathrm{P}-\mathrm{E}
$$

Où $\mathrm{Q}$ est l'écoulement annuel moyen, $\mathrm{P}$ la précipitation annuelle moyenne et $\mathrm{E}$ les pertes en eau annuelles moyennes. Les autres termes du bilan hydrologique, stockage/ déstockage à long terme, échanges souterrains, sont négligés. E est la somme de l'évapotranspiration réelle (ET) et de la sublimation du manteau neigeux (S). Favier et al. [2009]ont considéré que l'évapotranspiration est négligeable au dessus de l'altitude de $3000 \mathrm{~m}$. Au delà de ce seuil, les bassins versants sont abrupts, rocheux et la végétation absente au delà de quelques mètres de distance des cours d'eau. Dans ces zones, les pertes en eau par retour à l'atmosphère sont donc principalement dues à la sublimation du manteau neigeux hivernal. Nous avons fait l'hypothèse que le terme E peut être approché par le terme de sublimation sur l'ensemble de la zone d'étude.

La sublimation est calculée comme une fraction de la précipitation (équation 3) :

$$
\mathrm{E}=\mathrm{P} \times \mathrm{R} \times \mathrm{SCA}
$$

Où $\mathrm{R}$ est le rapport de la sublimation sur l'ablation totale déterminé expérimentalement et SCA est la surface enneigée (Snow Cover Area) annuelle moyenne, déterminée grâce aux images MODIS. Elle exprime en pourcentage pour chaque bassin versant l'extension spatiale de la couverture neigeuse.

Les résultats (Tableau 2) montrent que pour les bassins versants d'altitude pour lesquels notre hypothèse est la plus pertinente, l'introduction d'un terme de sublimation estimé assez grossièrement n'entraîne pas de dépassement de la valeur maximale physiquement acceptable du

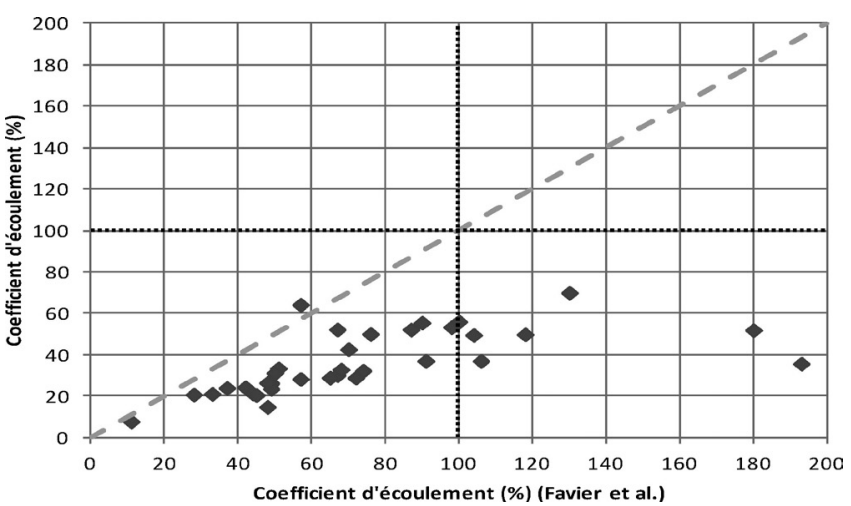

Figure 5 : Comparaison des coefficients d'écoulement obtenus avec la méthode de Valéry [2010] et ceux de Favier et al. [2009]

Tableau $2:$ Les 10 bassins versants les plus exposés à la couverture neigeuse. P est la précipitation moyenne annuelle de bassin en mm/an, $Q$ l'écoulement moyen annuel en mm/an.

\begin{tabular}{|c|c|c|c|c|c|c|c|}
\hline Bassin versant & $\begin{array}{c}\text { Surface } \\
\mathbf{( k m}^{2}\end{array}$ & $\begin{array}{c}\text { Altitude } \\
\mathbf{e x u t o i r e ~} \mathbf{( m )}\end{array}$ & $\mathbf{P}(\mathbf{m m})$ & $\begin{array}{c}\mathbf{Q} \\
\mathbf{( m m})\end{array}$ & $\mathbf{Q} / \mathbf{P}$ & $\mathbf{S C A}$ & $\mathbf{Q} / \mathbf{( P - E )}$ \\
\hline Rio Choapa en Cuncumen & 1115 & 1200 & 459 & 294 & $64 \%$ & $34 \%$ & $87 \%$ \\
\hline Rio la Laguna en salida embalse la Laguna & 558 & 3130 & 238 & 124 & $53 \%$ & $31 \%$ & $70 \%$ \\
\hline Rio Tascadero en Desembocadura & 236 & 1370 & 434 & 227 & $52 \%$ & $29 \%$ & $67 \%$ \\
\hline Rio Grande en Las Ramadas & 691 & 1380 & 446 & 224 & $50 \%$ & $29 \%$ & $64 \%$ \\
\hline Rio Cuncumen antes Bocatema de Canales & 225 & 1360 & 443 & 168 & $37 \%$ & $29 \%$ & $48 \%$ \\
\hline Rio Hurtado en San Agustin & 670 & 2035 & 261 & 146 & $56 \%$ & $28 \%$ & $71 \%$ \\
\hline Rio Cochiguaz en El Penon & 679 & 1360 & 213 & 149 & $70 \%$ & $27 \%$ & $88 \%$ \\
\hline Rio Chalinga en La Palmilla & 242 & 800 & 388 & 143 & $36 \%$ & $25 \%$ & $44 \%$ \\
\hline Rio Mostazal en Cuestecita & 391 & 1250 & 383 & 161 & $42 \%$ & $25 \%$ & $52 \%$ \\
\hline Estero Derecho Alcoguaz & 421 & 1645 & 221 & 112 & $50 \%$ & $24 \%$ & $62 \%$ \\
\hline Rio Cambarbala en Ramadillas & 190 & 1430 & 326 & 166 & $52 \%$ & $20 \%$ & $61 \%$ \\
\hline
\end{tabular}


ratio $\mathrm{Q} /(\mathrm{P}-\mathrm{E})$ et donne des valeurs relativement proches de 1. Pour les autres bassins, moins soumis à l'enneigement, l'hypothèse n'est plus vraisemblable puisque les pertes par évapotranspiration sont alors dominantes. Ce résultat semble confirmer la vraisemblance du champ de précipitation testé et montre sa capacité à améliorer le bouclage du bilan hydrologique sur les bassins versants en altitude du Norte Chico.

\section{CONCLUSIONS ET PERSPECTIVES}

L'application d'une méthodologie de validation à trois niveaux distincts a permis de révéler les bénéfices apportés à la connaissance hydrologique de la région du Norte Chico par une méthode innovante d'interpolation spatiale de la précipitation. L'extrême variabilité spatiale et temporelle des précipitations sur la région et le manque de données d'observations en altitude posent des défis considérables quant-à la connaissance précise de l'hydrologie de la zone d'étude et constituent deux sources considérables d'incertitudes concernant le calcul du champ de précipitation. Parmi les perspectives d'amélioration envisagées figurent l'application de la méthode d'interpolation à la variable température et l'intégration des données supplémentaires suite à l'équipement récent de certains points en altitude. L'intégration de cette méthode d'interpolation dans un modèle hydrologique global reste à tester. Plus modestement, le calul du ratio $\mathrm{Q} /(\mathrm{P}-\mathrm{E})$ pourrait être réalisé au pas de temps annuel. Enfin, une approche plus directe du calcul des pertes par retour à l'atmosphère est envisagée pour compléter le bouclage du bilan hydrologique dans les bassins versants les plus à l'aval.

\section{REFERENCES AND CITATIONS}

FaVier V., Et AL. (2009) - Interpreting discrepancies between discharge and precipitation in high-altitude area of Chile's Norte Chico region (26-32 degrees S). Water Resources Research 45

LLOYD C.D. (2005) - Assessing the effect of integrating elevation data into the estimation of monthly precipitation in Great Britain Journal of Hydrology. 308(1-4) 128-150

VAlery A. (2010) - Thèse de Doctorat, Modélisation précipitations-débit sous influence nivale. Elaboration d'un module neige et évaluation sur 380 bassins versants

Viviroli D. And R. Weingartner (2004) - The hydrological significance of mountains : from regional to global scale. Hydrology and Earth System Sciences. 8(6) 1016-1029 